\title{
A Case of Hyperparathyroidism Caused by Intrathyroidal Parathyroid Adenoma
}

\author{
Ki Ju Cho', Sang-Wook Park ${ }^{1}$, Seongjun Won ${ }^{1}$, Jin Pyeong Kim ${ }^{1,2}$ and \\ Jung Je Park ${ }^{1,2}$
}

Department of Otorhinolaryngology-Head \& Neck Surgery, Gyeongsang National University Hospital, Gyeongsang National University School of Medicine, Institute of Health Science, Gyeongsang National University School of Medicine ${ }^{2}$, Jinju, Korea

Hyperparathyroidism is a common endocrine disorder, but intrathyroidal parathyroid adenoma is extremely rare. We report a on a case of hyperparathyroidism caused by intrathyroidal parathyroid adenoma. This case is important in that it affects an effective test for the diagnosis of intrathyroidal parathyroid adenoma, and shows the decision of the treatment process and the results.

Key Words: Hyperparathyroidism, Ectopic parathyroid, Parathyroid adenoma, Intrathyroidal parathyroid adenoma

\section{Introduction}

The parathyroid gland is mainly located behind the thyroid gland, but it is sometimes found in a variety of places, from carotid bifurcation to pericardium. Ectopic parathyroid gland occurs when the parathyroid gland is not in its proper location." Most literature reports it is caused by embryologically abnormal migration. ${ }^{2)}$ An intrathyroidal parathyroid adenoma is one way an ectopic parathyroid gland is manifested. This is rare, and its incidence is reported to be about $1.3 \%$ to $6.7 \%{ }^{3)}$ We report on a case with suspected parathyroid adenoma before surgery, but the finally confirmed intrathyroidal parathyroid adenoma through pathological evaluation during and following the surgery.

\section{Case Report}

A 48-year-old women was referred for a left thyroid nodule, which was accidentally discovered, and pri- mary hyperparathyroidism with hypercalcemia. On admission, she did not complain of any special symptoms related to hypercalcemia. Vocal cord movement was normal on laryngeal endoscopic exam. Serum calcium was elevated to $11.1 \mathrm{mg} / \mathrm{dL}$ (reference range: 8.6-10.2) and serum phosphate level was decreased to $2.5 \mathrm{mg} / \mathrm{dL}$ (reference range: $2.7-4.5$ ). Intact parathyroid hormone (iPTH) level was elevated to 182.8 $\mathrm{pg} / \mathrm{mL}$ (reference range: 10-55), but thyroid function tests were within normal range. Bone densitometry was confirmed in the normal range. Urine metanephrine, calcitonin, and pituitary function tests were performed for 24 hours to confirm normal findings, and multiple endocrine neoplasms were excluded. Computed tomography (CT) of the neck. The scan with contrast enhancement shows a heterogeneous soft tissue mass, clearly defined, within the left thyroid lobe (Fig. 1). Neck ultrasonography (US) showed that the thyroid gland was normal and a hypoechoic nodule measuring $1.77 \times 1.13 \times 3.32 \mathrm{~cm}$ in the left upper pole of thyroid (Fig. 2). The lesion was embedded on the

Received December 21, 2020 / Revised January 15, 2021 / Accepted January 28, 2021

Correspondence: Jung Je Park, MD, PhD, Department of Otorhinolaryngology-Head \& Neck Surgery, Gyeongsang National University Hospital, Gyeongsang National University School of Medicine, 9, Gangnam-ro, Jinju 52727, Korea

Tel: 82-55-750-8178, Fax: 82-55-759-0613, E-mail: capetown@hanmail.net 


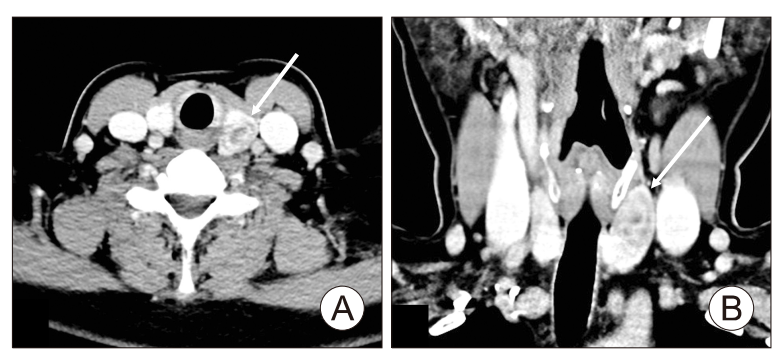

Fig. 1. Computed tomography of the neck. The scan with contrast enhancement shows a heterogeneous soft tissue mass, clearly defined, within the left thyroid lobe (white arrows). (A) Axial view. (B) Coronal view.

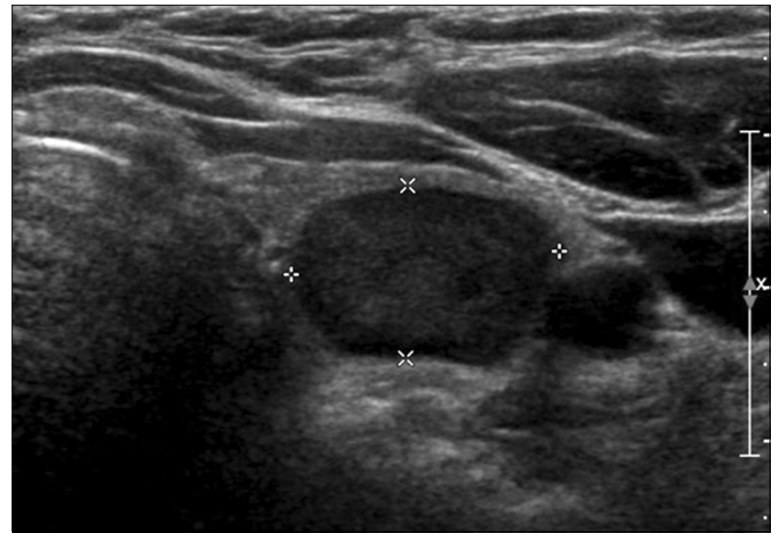

Fig. 2. Thyroid ultrasonography showed $1.77 \times 1.13 \times 3.32$ $\mathrm{cm}$ hypoechoic nodule in left thyroid gland.

upper left thyroid. Fine needle aspiration (FNA) of the lesion was additionally performed. Atypical cells were identified in US-guided FNA cytological test. In the Technesium (Tc)-99m sestamibi parathyroid scan, Early and delayed scintigrams reveal focal accumulation of increased radiotracer uptake in the left lobe of the thyroid (Fig. 3).

By combining the above findings, left parathyroid adenoma was suspected and left parathyroidectomy was planned. The left lower parathyroid gland was identified and removed during surgery, but the left upper parathyroid gland was not observed. Intraoperatively, the tumor was touched and felt in the left upper lobe of the thyroid gland, but the boundary of the tumor could not be confirmed. So, left thyroidectomy was performed under suspicion of intrathyroidal parathyroid adenoma, and the left recurrent laryngeal nerve was preserved. The size of the left thyroid lobe was $4.5 \times 2.4 \times 2 \mathrm{~cm}$ (Fig. 4). In the frozen section exami-

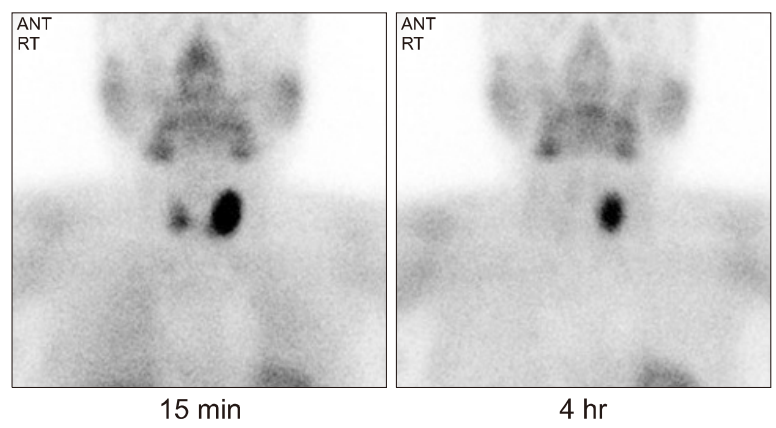

Fig. 3. Technesium (Tc)-99m sestamibi parathyroid scan. Early and delayed scintigrams reveal focal accumulation of increased radiotracer uptake in the left lobe of the thyroid.
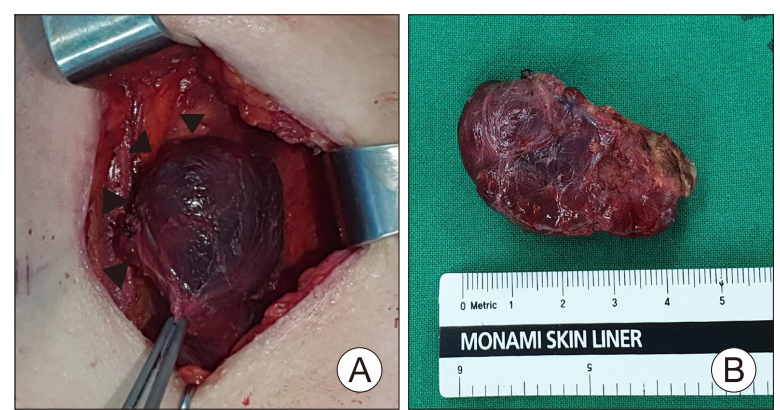

Fig. 4. Gross photo of specimen. (A) Exposed left side of thyroid tissue (arrowheads). (B) The gross pathology specimen of left thyroid.

nation performed during surgery, the parathyroid adenoma (size $1.7 \times 1.1 \times 3.3 \mathrm{~cm}$ ) was found inside the left thyroid lobe. Normal parathyroid tissue was identified in the resected lower parathyroid gland. The iPTH was measured intraoperatively, and it was found to decreased from $140 \mathrm{pg} / \mathrm{mL}$ before incision to 26.69 $\mathrm{pg} / \mathrm{mL}$ following the left thyroid resection. ${ }^{4}$ In pathology, biopsies revealed an intrathyroidal parathyroid adenoma within the normal thyroid gland, with colloid follicles on the periphery. This was separated from fibrous tissue (Fig. 5A). This was described as an encapsulated intrathyroidal parathyroid adenoma with proliferation of parathyroid cells (Fig. 5B). The histological diagnosis was supported by immunohistochemistry, and the tumor cells presented with strongly diffuse positivity for GATA3 (Fig. 5C) and no staining for TTF1 (Fig. 5D). The results showed a strong positive for GATA3, confirming that this was a parathyroid tumor. They were also negative for TTF1, showing that thyroid carcinoma could be excluded. 

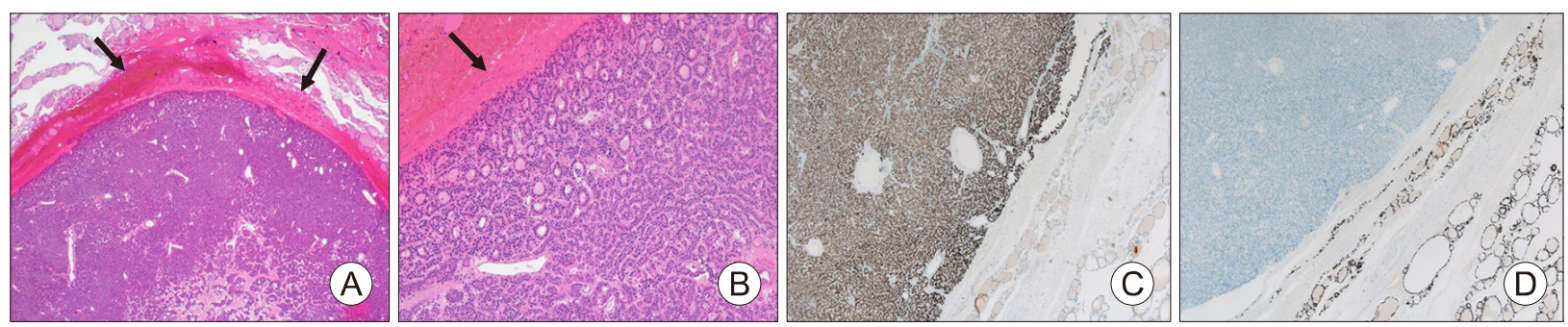

Fig. 5. Histopathologic findings of left thyroid gland. Microscopic findings show well-encapsulated parathyroid adenoma inside the normal thyroid tissue. (A) Hematoxylin and Eosin (H\&E) stain, $\times 40$, (B) H\&E stain, $\times 100$ (arrows). The tumor cells showed diffuse strong positivity for GATA3, but absence of staining for TTF1. (C) GATA3 immune stain, $\times 40$. (D) TTF1 immune stain, $\times 40$.

There were no side effects such as vocal cord paralysis following surgery, and the iPTH on the first day following surgery decreased to a normal range of 5.89 $\mathrm{pg} / \mathrm{mL}$ (reference range: 10-55). The calcium level was $9.4 \mathrm{mg} / \mathrm{dL}$ (reference range: 8.6-10.2). Seven days following surgery, the calcium level was maintained within the normal range of $9.2 \mathrm{mg} / \mathrm{dL}$ (reference range: 8.6-10.2). She underwent outpatient follow-up at the endocrinology department without any special side effects or problems after 4 months of surgery.

\section{Discussion}

Hyperparathyroidism can manifest as symptoms of anorexia, mental state change, depression, fatigue, nausea, vomiting, peptic ulcer disease, constipation, polyuria, renal stones, cardiac arrhythmias, and a shortened QT interval. ${ }^{5)}$ There are three forms of hyperparathyroidism: primary, secondary, and tertiary. Primary hyperparathyroidism is usually the result of elevated levels of parathyroid hormone and calcium. The patient has a generally decreased phosphate level. The secondary form occurs when resistance to the action of parathyroid hormones develops because of kidney failure or vitamin $D$ deficiency, leading to parathyroid hyperplasia. The tertiary form appears when patients with secondary form develop autonomic activity of the parathyroid gland. It is known that about $80 \%$ of hyperparathyroidism is associated with a solitary adenoma. Primary hyperparathyroidism is considered the second most common cause of predominant hypercalcemia in postmenopausal women. The treatment of choice for symptomatic hypercalcemia is parathyroidectomy. ${ }^{5)}$ In our case, there was no specific symptom of hyperparathyroidism, and this is a primary hyperparathyroidism with increased levels of parathyroid hormone and calcium.

Usually, there are three to four parathyroid glands including superior and inferior. The superior parathyroid gland derives from the fourth branchial arch. The inferior parathyroid gland, like the thymus, derives from the third branchial arch. It has been hypothesized that an intrathyroidal parathyroid gland is formed when the parathyroid tissue is trapped between the central and lateral lobes of the thyroid gland during their fusion. ${ }^{2,6)}$ Ectopic parathyroid glands occur more often in the inferior gland than in the upper gland because of the differentiation of embryonic development of the parathyroid gland. The inferior parathyroid gland descends through the neck and down the thymus along an embryonic long virtual tract, and the superior parathyroid gland descends through a shorter length compared to the inferior parathyroid gland. ${ }^{1)}$ Our case was rarer because it was an intrathyroidal parathyroid adenoma in the superior parathyroid gland.

US of the thyroid gland is usually done in patients with primary hyperparathyroidism. US detects parathyroid hyperplasia, parathyroid adenoma, and parathyroid carcinoma as hypoechoic nodules that can appear as cyst or calcifications. But normal and ectopic parathyroid glands within the mediastinum cannot be seen on US. ${ }^{7)}$ CT is a good additional test to complement these limitations. ${ }^{8)}$

For localization of parathyroid lesions, the difference in Tc-99m sestamibi washout between the thyroid and parathyroid glands is the most important. This is 
because parathyroid tissue absorbs more Tc-99m sestamibi per gram of tissue than does the thyroid tissue. In our case, after 15 minutes and four hours, the intake of Tc-99m sestamibi remained for a long time in the left lobe of the thyroid gland. This intake was increased, which can be predictive of the location of the lesion and the parathyroid tissue., ${ }^{7,9)}$

However, if the parathyroid adenoma cannot be found at the surgical field even after carefully surgical filed examination, suspected-sided thyroid lobectomy should be performed during the operation with the ectopic parathyroid gland in mind to remove the undetected lesion.

On pathological examination, intrathyroidal parathyroid adenoma can be mistaken for follicular thyroid carcinoma or medullary thyroid carcinoma. Therefore, it is believed that parathyroid hormone immunocytochemical staining is important in diagnosing of intrathyroidal parathyroid adenoma. ${ }^{10)}$

In conclusion, hyperparathyroidism is a common endocrine disorder, but intrathyroidal parathyroid adenoma is rare. This case is an intrathyroidal parathyroid adenoma in the superior parathyroid gland, which is more rare. In order to distinguish the cause of hyperparathyroidism, such as US and CT can be helpful. These can be useful tools in interpreting the Tc-99m sestamibi scans of the thyroid and parathyroid glands. Knowing the pathologic findings following surgery can be of benefit in clarifying the diagnosis. Also, if the parathyroid adenoma is palpable during surgery, but is not clearly distinguished, a intrathyroidal parathyroid adenoma should be suspected.

\section{Conflicts of Interest}

No potential conflict of interest relevant to this ar- ticle was reported

\section{Orcid}

Ki Ju Cho: https://orcid.org/0000-0002-1414-6655

Sang-Wook Park: https://orcid.org/0000-0002-7579-2327

Seongjun Won: https://orcid.org/0000-0002-8088-2554

Jin Pyeong Kim: https://orcid.org/0000-0001-6137-8232

Jung Je Park: https://orcid.org/0000-0001-6325-0398

\section{References}

1) Phitayakorn R, McHenry CR. Incidence and location of ectopic abnormal parathyroid glands. Am J Surg 2006;191(3):418-23.

2) Policeni BA, Smoker WR, Reede DL. Anatomy and embryology of the thyroid and parathyroid glands. Semin Ultrasound CT MR 2012;33(2):104-14.

3) Goodman A, Politz D, Lopez J, Norman J. Intrathyroid parathyroid adenoma: incidence and location--the case against thyroid lobectomy. Otolaryngol Head Neck Surg 2011;144(6): 867-71.

4) Paek SH, Kim SJ, Choi JY, Lee KE. Clinical usefulness of intraoperative parathyroid hormone monitoring for primary hyperparathyroidism. Ann Surg Treat Res 2018;94(2):69-73.

5) Taniegra ED. Hyperparathyroidism. Am Fam Physician 2004; 69(2):333-9.

6) Rossi ED, Mule A, Zannoni GF, Fadda G. Asymptomatic intrathyroidal parathyroid adenoma. Report of a case with a cytologic differential diagnosis including thyroid neoplasms. Acta Cytol 2004;48(3):437-40.

7) Steward DL, Danielson GP, Afman CE, Welge JA. Parathyroid adenoma localization: surgeon-performed ultrasound versus sestamibi. Laryngoscope 2006;116(8):1380-4.

8) Khati N, Adamson T, Johnson KS, Hill MC. Ultrasound of the thyroid and parathyroid glands. Ultrasound Q 2003;19(4): 162-76.

9) Fernandez KL, Turer P, Spiegler EJ, Singer JA. The use of sestamibi imaging in parathyroid hyperplasia. Clin Nucl Med 2002;27(12):865-7.

10) Feliciano DV. Parathyroid pathology in an intrathyroidal position. Am J Surg 1992;164(5):496-500. 